\title{
Equilibria with vector-valued utilities and preference information. The analysis of a mixed duopoly.
}

\author{
A.M. Mármol, L. Monroy† M.A. Caraballo
}

\begin{abstract}
This paper deals with the equilibria of games when the agents have multiple objectives and, therefore, their utilities cannot be represented by a single value, but by a vector containing the various dimensions of the utility. Our approach allows the incorporation of partial information about the preferences of the agents into the model, and permits the identification of the set of equilibria in accordance with this information. We also propose an additional conservative criterion which can be applied in this framework in order to predict the results of interaction.

The potential application of the theoretical results is shown with an analysis of a mixed oligopoly in which the agents value additional objectives other than their own benefit. These objectives are related to social welfare and to the profit of the industry. The flexibility of our approach provides a general theoretical framework for the analysis of a wide range of strategic economic models.
\end{abstract}

Keywords: non-cooperative games, vector-valued utility, equilibria, preference information, mixed duopoly.

*Corresponding author. Dpto. de Economía Aplicada III and IMUS. Universidad de Sevilla, Spain. amarmol@us.es.

${ }^{\dagger}$ Dpto. de Economía Aplicada III and IMUS. Universidad de Sevilla, Spain. lmonroy@us.es.

${ }_{\ddagger}^{\ddagger}$ Dpto. de Economía e Historia Económica, Universidad de Sevilla, Spain. mcaraba@us.es.

§Dpto. de Economía Aplicada III, Universidad de Sevilla, Spain. azapata@us.es. 


\section{Introduction}

The theory of games with vector payoffs is concerned with situations in which a number of players must take into account several objectives, each of which depends on the decision of all players. This type of game was introduced by Blackwell (1956). Subsequently, Shapley (1959) presented the natural extensions of the concept of Nash equilibrium, (Nash 1951), for two-person zero-sum finite games with vector payoffs: strong equilibria and weak equilibria, which are the basic concepts in multiobjective games. Among the authors who have studied the existence of equilibria for this kind of game are Zeleny (1975), Corley (1985), and Borm et al. (1988). For general $n$-person multi-objective games, the first results on the existence of equilibria were established in Zhao (1991). Interesting work on the topic includes Voorneveld et al. (1999), Bade (2005), and Patriche (2014).

The present paper is also devoted to the analysis of equilibria of $n$-person noncooperative games where the payoffs of the agents are multi-dimensional. We call them games with vector-valued utilities. These games represent situations in which the preferences of the agents on the results of the interactions are incomplete. In the existing literature on models with incomplete preferences, the two classic references are Aumann (1962) and Bewley (1986). More recently, this decisional framework has been studied from various viewpoints. In particular, certain authors have established a formal connection between incomplete preferences and multi-objective decision-making under certainty and risk (Ok, 2002; Dubra et al., 2004; Sagi, 2006). The fundamental difficulty is the impossibility of representing incomplete preferences in terms of utility functions, and hence the application range remains limited. This is the main cause of the scarcity of results in this line of research. However, as shown in Ok (2002), under certain not particularly restrictive hypotheses, incomplete preferences can be represented by means of vector-valued utility functions. This approach causes no loss of information and enables these situations to be studied from an analytical standpoint by using the well-developed theory of vector optimization in the operations research literature. The literature on incomplete preferences mainly deals with issues of individual choice, and only a few papers address non-cooperative models of interaction between agents with incomplete preferences. Some exceptions of note are found in Shafer and Sonnenschein (1975), Bade (2005), and Park (2015).

As mentioned in Bade (2005), one argument for studying games with vectorvalued utilities is the possibility of addressing cases in which agents do have weighted utilities over multiple criteria but the modeler, as an outside observer, remains 
unaware of agents' weights. In these situations, the set of equilibria of the game modeled as a vector-valued game will always include the equilibria of the actual game.

An alternative interpretation of the equilibria in this model is that the agents are uncertain about their own weights and they will only deviate when the deviation gain is positive for every possible weight vector. Nevertheless, in either interpretation, it is also possible that the agents' weights are known to belong to certain subsets, in which case the incorporation of this information into the model should lead to predictions that are better adjusted.

In the present paper, we initially adopt the formal framework in Bade (2005) to model $n$-person games with incomplete preferences that can be represented by a vector of utility functions. In Bade's paper, the equilibria for these games are characterized in terms of the equilibria of weighted games under reasonable concavity assumptions. Her results are then applied to identify the sets of equilibria in several examples from oligopoly theory. However, the set of equilibria of games with incomplete preferences may contain a large number of strategy profiles, some of which may not represent realistic predictions. For this reason, the first goal of our research is to present a procedure to obtain a number of refinements of the set of equilibria based on partial preference information. Thus, we can study which kind of predictions are derived from the information available without requiring additional assumptions about the utility functions of the agents.

In order to deal with partial information, we need to rely on the weak extension of the standard concept of the Nash equilibrium (see Shapley, 1959; Wang, 1993; Voorneveld et al., 1999). The adoption of this extension is not, in general, a drawback, since the sets of strong equilibria and weak equilibria often coincide. In fact, under certain concavity assumptions, the two concepts of equilibrium coincide. When they do not, the difference usually lies on boundary points.

A first interesting result is the characterization of equilibria in terms of the reaction functions of the components of the utility. This result is relevant from an operational point of view, and will recursively be applied throughout the paper for the identification of equilibria.

We assume that the underlying incomplete preferences of the agents can be represented by weighted additive value functions where weights may be interpreted as the relative importance that the agents assign to the components of their vector utility functions. The equilibria of the game are then identified with the equilibria of the corresponding weighted games. In this framework, information about preferences 
is formalized by means of sets of weights which, in general, are different for each agent. A central result is established which identifies the set of equilibria of the game with partial preference information with the set of equilibria of a transformed vector-valued game.

The inclusion of agents' preferences into the model through admissible weights provides the equilibria in accordance with these preferences. However, it is often the case that the agents, even when they admit that a certain set of weights are possible, might also exhibit a conservative attitude with respect to the results they will eventually achieve. Empirical evidence that agents are more uncertainty-averse than uncertainty-loving can be found, for instance, in Wakker (2001). More recently, Kozhan and Salmon (2009) found significant evidence of uncertainty aversion in the foreign exchange market.

Thus, we apply a worst-case analysis based on the well-known maxmin criterion proposed by Wald (1950), and adapted to partial preference information (see also Gilboa and Schmeidler, 1989). We propose a refinement based on a rule by which the agents select their decisions by maximizing the worst evaluation from among those provided by the feasible weights in their information sets.

A second goal of this paper is to show the potential of this analysis for the study of non-cooperative economic models. The theoretical results herein developed have been applied to a relevant type of decision-making process with interacting agents. We have extended the analysis of standard oligopoly models from firms that only consider the maximization of their own profits to a more realistic situation where firms incorporate additional goals.

We have focused on mixed oligopoly models. Traditionally, these models are those that consider private firms that are profit maximizers and public firms with social goals. Since the seminal paper of Merrill and Schneider (1966) appeared, there has been a growing literature concerning several aspects and implications of mixed duopolies in the markets (see De Fraja and Del Bono, 1989, 1990) and the references therein). Nevertheless, the literature on the topic usually excludes profit maximization from the goals of the social firms.

Our approach is more flexible and permits the analysis of firms with social objectives which do not completely abandon the pursuit of maximum profits that ensures their permanence in the market. We specifically analyze the case of mixed duopoly under various assumptions about the firms' objectives and present the results and interpretations regarding the sets of equilibria.

The following notation will be used. Let $\mathbb{R}\left(\mathbb{R}_{+}\right)$denote the set of all (non- 
negative) real numbers and let $\mathbb{R}^{k}\left(\mathbb{R}_{+}^{k}\right)$ be the $k$-fold Cartesian product of $\mathbb{R}\left(\mathbb{R}_{+}\right)$. The origin of $\mathbb{R}^{k}$ is $0^{k}$ and $1^{k}$ is a $k$-dimensional vector with components equal to one. We use conventional notation for the comparison of vectors: $x \geqq y$ means that $x_{i} \geq y_{i}$ for all $i=1, \ldots, k ; x \geq y$ indicates that $x \geq y$ and $x \neq y$; and $x>y$ indicates that $x_{i}>y_{i}$ for all $i=1, \ldots, k$. By $x \cdot y$ we denote the scalar product of vectors $x, y \in \mathbb{R}^{k}$, that is, $x \cdot y=\sum_{i=1}^{k} x_{i} y_{i}$.

The rest of the paper is organized as follows. In Section 2 the concepts of equilibria and weak equilibria for $n$-person games with vector-valued utility functions are stated and their relationship with the equilibria of weighted games is investigated. In Section 3, we present refinements of the equilibria by incorporating information about the preferences of the agents into the model. Section 4 is devoted to the analysis of a mixed oligopoly in which the firms pursue additional goals to those of maximizing profits. Section 5 is devoted to setting out the conclusions. Proofs are contained in an Appendix.

\section{Equilibria in games with vector-valued utilities}

This section is devoted to the study of the set of equilibria of $n$-person games with vector-valued utility functions.

A vector-valued normal-form game is represented by $G=\left\{\left(A^{i}, u^{i}\right)_{i \in N}\right\}$, where $N=\{1, \ldots, n\}$ is the set of agents, $A^{i}$ is the set of strategies that agent $i \in$ $N$ can adopt and the mapping $u^{i}: \times_{i \in N} A^{i} \rightarrow \mathbb{R}^{s^{i}}$ is the vector-valued utility function of agent $i, u^{i}:=\left(u_{1}^{i}, \ldots, u_{s^{i}}^{i}\right)$, where $s^{i}$ is the number of components of the utility function of agent $i$. We denote by $J^{i}=\left\{1, \ldots, s^{i}\right\}$ the set of indices of such components. A profile of strategies, $a=\left(a^{1}, \ldots, a^{n}\right)$, with $a^{i} \in A^{i}$, for a game $G$ can be written as $a=\left(a^{i}, a^{-i}\right)$, where $a^{i}$ is a strategy of agent $i$, and $a^{-i}=\left(a^{1}, \ldots, a^{i-1}, a^{i+1}, \ldots, a^{n}\right)$ stands for the strategy combination of all players except player $i$.

The following definitions are extensions of the concept of Nash equilibrium for these games with vector-valued utilities. They were introduced by Shapley (1959) for finite two-person zero-sum games.

Definition 2.1. An action profile $a^{*}=\left(a^{* 1}, \ldots, a^{* n}\right)$ is a weak equilibrium for the game with vector-valued utilities $G=\left\{\left(A^{i}, u^{i}\right)_{i \in N}\right\}$ if $\nexists i \in N$ with $a^{i} \in A^{i}$ such that $u^{i}\left(a^{i}, a^{*-i}\right)>u^{i}\left(a^{*}\right)$.

The set of all weak equilibria of game $G$ is denoted by $E^{w}(G)$. 
A weak equilibrium does not necessarily yield the best overall result for all agents. However, in a weak equilibrium, agents cannot improve all the components of their utilities by deviating independently.

The other extension of the concept of Nash equilibrium is stronger than the first extension:

Definition 2.2. An action profile $a^{*}=\left(a^{* 1}, \ldots, a^{* n}\right)$ is an equilibrium for the game with vector-valued utilities $G=\left\{\left(A^{i}, u^{i}\right)_{i \in N}\right\}$ if $\nexists i \in N$ with $a^{i} \in A^{i}$ such that $u^{i}\left(a^{i}, a^{*-i}\right) \geq u^{i}\left(a^{* i}, a^{*-i}\right)$.

The set of all equilibria of game $G$ is denoted by $E(G)$.

Note that both Definition 2.1 and Definition 2.2 reduce to the standard definition of Nash equilibrium when the utility function of each agent is a real-valued function.

The set of weak equilibria and the set of equilibria only slightly differ. Under certain concavity conditions ${ }^{1}$, which often hold in applications, both sets coincide. When these sets are different, the set of equilibria can exclude some of the boundary points, which are included in the set of weak equilibria.

Let $r_{j}^{i}$ denote the correspondence of best response of agent $i$ in relation to the $j$-th utility component. Note that for each $a^{-i} \in A^{-i}, r_{j}^{i}\left(a^{-i}\right) \subseteq A^{i}$. The best response of an agent to the actions of all the others may not exist and when it does, it is possibly not a singleton. However, under certain conditions on the set of strategies and on the concavity of the utility functions, it is possible to identify the set of equilibria of the game with multi-dimensional utilities in terms of the reaction function of the components as shown in the following result.

Theorem 2.3. If $A^{i}$ is a non-empty convex compact subset $A^{i} \subseteq \mathbb{R}$ for all $i \in N$, and $u_{j}^{i}$ is continuous and strictly concave in its own action for each $j \in J^{i}$, then the set of equilibria of the game with vector-valued utilities $G=\left\{\left(A^{i}, u^{i}\right)_{i \in N}\right\}$ is

$$
E(G)=\left\{\left(a^{1}, \ldots, a^{n}\right) \in \times_{i \in N} A^{i}: \underline{r}^{i}\left(a^{-i}\right) \leq a^{i} \leq \bar{r}^{i}\left(a^{-i}\right), i \in N\right\},
$$

where $\underline{r}^{i}\left(a^{-i}\right)=\min _{j \in J^{i}} r_{j}^{i}\left(a^{-i}\right)$, and $\bar{r}^{i}\left(a^{-i}\right)=\max _{j \in J^{i}} r_{j}^{i}\left(a^{-i}\right)$.

Under the hypothesis of strict concavity of the components of the utility, the set of equilibria and the set of weak equilibria coincide. This hypothesis can be relaxed to concavity, giving rise to a similar result which characterizes the set of

\footnotetext{
${ }^{1}$ For instance, a sufficient condition for the two sets to coincide is that the sets $A^{i}$ are non-empty convex subsets of a finite dimensional space, and the functions $u_{j}^{i}$ are strictly concave in $a^{i}$.
} 
weak equilibria in terms of the minimum and of the maximum of the best responses, by slightly modifying the definition of $\underline{r}_{j}^{i}\left(a^{-i}\right)$ and $\underline{r}_{j}^{i}\left(a^{-i}\right)$ when some of the best responses are not a singleton.

\subsection{Weighted games and equilibria}

In a game with vector-valued utilities, the preferences of the agents on the profiles of strategies are incomplete. The literature on vector-valued optimization problems has focused on the case where the preferences of the agents are represented by additive value functions since this type of function permits the representation of preferences under not very restrictive assumptions (see Keeney and Raiffa, 1976). In this paper, we also assume that the preferences of each agent can be represented by a weighted additive value function. The weights represent trade-offs between the various components of the utility, and can be interpreted as the relative importance that this agent assigns to the components of his/her vector-utility function.

When complete information about the importance of the components is available, then the preferences of each agent are represented by a value function determined by a unique vector of weights, and the problem of equilibria identification in the vector-valued game is reduced to the identification of equilibria in scalar games.

Formally, given a vector of preference weights for agent $i, \lambda^{i} \in \Delta^{s^{i}}=\left\{\lambda^{i} \in \mathbb{R}^{s^{i}}\right.$ : $\left.\sum_{j=1}^{s^{i}} \lambda_{j}^{i}=1, \lambda_{j}^{i} \geq 0\right\}$, the value of the action profile $a \in \times_{i=1}^{n} A^{i}$ for agent $i$ is given by $\sum_{j=1}^{s^{i}} \lambda_{j}^{i} u_{j}^{i}(a)$. Consider $\Delta=\times_{i=1}^{n} \Delta^{s^{i}}$. For $\lambda \in \Delta$, we define the normal-form weighted game $G_{\lambda}=\left\{\left(A^{i}, v_{\lambda}^{i}\right)_{i \in N}\right\}$ with

$$
v_{\lambda}^{i}(a)=\sum_{j=1}^{s^{i}} \lambda_{j}^{i} u_{j}^{i}(a) .
$$

The concept of equilibrium for the scalar game $G_{\lambda}$ is the following.

Definition 2.4. For $\lambda \in \Delta$, an action profile $a^{*}=\left(a^{* 1}, \ldots, a^{* n}\right)$ is a Nash equilibrium for the game $G_{\lambda}$ if $\nexists i \in N$ with $a^{i} \in A^{i}$ such that $v_{\lambda}\left(a^{i}, a^{*-i}\right)>v_{\lambda}\left(a^{*}\right)$.

$E\left(G_{\lambda}\right)$ denotes the set of Nash equilibria for the weighted game $G_{\lambda}=\left\{\left(A^{i}, v_{\lambda}^{i}\right)_{i \in N}\right\}$.

Note that we can write $v_{\lambda}^{i}(a)=\lambda^{i} \cdot u^{i}(a)$. Definition 2.4 can alternatively be written as: $a^{*}$ is a Nash equilibrium for the game $G_{\lambda}$ if for each $i \in N, \lambda^{i} \cdot u^{i}\left(a^{*}\right) \geq$ $\lambda^{i} \cdot u^{i}\left(a^{i}, a^{*-i}\right)$, for all $a^{i} \in A^{i}$.

In vector-valued games, if the agents consider all the possible values of weights, then all the additive representations of preferences are taken into account. Bade 
(2005) establishes the relationship between the set of equilibria of the game and the set of equilibria of the weighted games with positive weights and with non-negative weights. She shows that if the utility functions of all players are concave in their own actions, and all action spaces are convex, then

$$
\cup\left\{E\left(G_{\lambda}\right): \lambda \in \Delta^{+}\right\} \subseteq E(G) \subseteq \cup\left\{E\left(G_{\lambda}\right): \lambda \in \Delta\right\} .
$$

where $\Delta^{+}=\left\{\lambda \in \Delta: \lambda_{j}^{i}>0, i \in N, j \in J^{i}\right\}$.

This result provides an upper and a lower bound on the set of all equilibria of the vector-valued game. However, these two sets that bound the set of equilibria, seldom differ. Indeed, if component-wise strict concavity is assumed, the three sets coincide.

On the other hand, Wang (1993) states that the equilibria of weighted games with non-negative weights are weak equilibria for the game with vector-valued utilities, that is,

$$
\cup\left\{E\left(G_{\lambda}\right): \lambda \in \Delta\right\} \subseteq E^{w}(G)
$$

This inclusion may be strict, that is, weak equilibria may exist that cannot be determined by using additive value functions. However, under concavity assumptions, the two sets coincide, as established in the following result.

Theorem 2.5. Let $G=\left\{\left(A^{i}, u^{i}\right)_{i \in N}\right\}$ be a game with vector-valued utilities such that each $A^{i}$ is a non-empty convex subset of a finite dimensional space and for each $i, u^{i}$ is concave in $a^{i}$, then

$$
\cup\left\{E\left(G_{\lambda}\right): \lambda \in \Delta\right\}=E^{w}(G) .
$$

This result constitutes a generalization of that established by Shapley (1959) for finite games with vector payoffs.

Moreover, when the components of $u^{i}$ are strictly concave functions, and the strategy sets are convex, then the set of equilibria coincides with the set of weak equilibria of all weighted games. This fact follows from our former result and from Theorem 3 in Bade (2005).

\section{$3 \quad$ Equilibria with preference information}

The identification of all equilibria is not always useful in operational terms, given the large number of equilibria that may exist. In addition, depending on the situation, 
not all equilibria are necessarily likely to be adopted by the agents. It is therefore valuable to study the appropriate refinements of the notion of equilibrium when additional considerations are incorporated.

An intermediate situation between the case of certainty about the preference weights and complete uncertainty is when certain information on these parameters is available. The information can be formalized by means of information sets, which in general are different for each agent. We explore the effects of including these information sets into the analysis with the aim of determining the equilibria which are in accordance with the agents' preference information.

Consider a subset of weights for each agent, $\Lambda^{i} \subseteq \Delta^{s^{i}}$, representing partial information on the preference weights of agent $i$. Denote by $\Lambda=\times_{i \in N} \Lambda^{i}$ the set containing the preference information of all the agents.

Definition 3.1. Let $G=\left\{\left(A^{i}, u^{i}\right)_{i \in N}\right\}$ be a game with vector-valued utilities. An action profile $a^{*}=\left(a^{* 1}, \ldots, a^{* n}\right)$ is an equilibrium for the game with preference information $\Lambda \subseteq \Delta^{s^{i}}$ if, for each $i \in N, \lambda^{i} \in \Lambda^{i}$ exists such that $v_{\lambda}^{i}\left(a^{*}\right) \geq v_{\lambda}^{i}\left(a^{i}, a^{*-i}\right)$ for all $a^{i} \in A^{i}$.

The set of equilibria of the game with preference information $\Lambda$ is denoted $E_{\Lambda}(G)$.

In other words, $a^{*}$ is an equilibrium for the game with preference information if $a^{*}$ is an equilibrium of the weighted game for some $\lambda \in \Lambda$.

In the absence of information, the information sets are $\Lambda^{i}=\Delta^{s^{i}}$ for all $i \in N$, and, under the assumptions of Theorem 2.5, the set of all weak equilibria is obtained. It is easy to prove that if $\Lambda^{\prime} \subset \Lambda$, then $E_{\Lambda^{\prime}}(G) \subseteq E_{\Lambda}(G)$. As a consequence, the refinement of the preference information yields a reduction of the set of equilibria. The limit case is when preference information reduces to a vector of weights for each agent. The preference relation is then complete and the equilibria correspond to the standard Nash equilibria for the real-valued utilities of the agents. Note that if the set of information only contains positive weights, then the equilibria with preference information are equilibria of the game with vector-valued utilities. That is, if $\Lambda \subseteq \Delta^{+}$, then $E_{\Lambda}(G) \subseteq E(G)$.

We are interested in certain special sets of weights, particularly those which can be described by linear relations. In these cases, the information sets are polyhedra, and thus they can be characterized by their extreme points.

The following two results are extensions to the more general framework of those results stated in Section 2. They permit us to incorporate results from games with complete preferences into the theory of games with incomplete preferences, and they 
allow the identification of the equilibria which are in accordance with the available preference information.

For $i \in N$, let $\Lambda^{i}$ be a polyhedron with $p_{i}$ extreme points $\left\{\bar{\lambda}(1), \ldots \bar{\lambda}\left(p_{i}\right)\right\}$, and let $B^{i}$ be the $p_{i} \times s_{i}$ matrix whose rows are the extreme points of $\Lambda^{i}$. For each $i \in N$, define a function, $v_{\Lambda}^{i}$, with values in $\mathbb{R}^{p_{i}}$, given by $v_{\Lambda}^{i}=B^{i} \cdot u^{i}$. Denote by $G_{\Lambda}=\left\{\left(A^{i}, v_{\Lambda}^{i}\right)_{i \in N}\right\}$ the transformed vector-valued game.

Proposition 3.2. Let $G=\left\{\left(A^{i}, u^{i}\right)_{i \in N}\right\}$ be a game with vector-valued utilities. Then

$$
E_{\Lambda}(G) \subseteq E^{w}\left(G_{\Lambda}\right)
$$

The following is a central result. It states that, under concavity conditions, the reverse is true, that is, it identifies the set of equilibria of the game with partial preference information with the set of weak equilibria of the transformed game.

Theorem 3.3. Let $G=\left\{\left(A^{i}, u^{i}\right)_{i \in N}\right\}$ be a game with vector-valued utilities such that each $A^{i}$ is a non-empty convex subset of a finite dimensional space and for each $i \in N, u^{i}$ is concave in $a^{i}$. Then,

$$
E_{\Lambda}(G)=E^{w}\left(G_{\Lambda}\right)
$$

\subsection{Conservative equilibria}

A conservative attitude is one of the most usual behaviors of the agents when assessing possible outcomes (see for instance, Wakker, 2001; Kozhan and Salmon, 2009). We now present an analysis of the equilibria of the game with vector-valued utilities that can be appropriate when the agents are averse to the uncertainty underlying the weights assigned to the components of the utility. We therefore assume that the agents' assessment of the results is based on the worst-case scenario, and by adopting a conservative attitude, the agents seek to guarantee the best results from among the worst expectations.

Thus, we consider a conservative value function. Each agent $i$ evaluates an action profile $a$ as the minimum weighted value among all the feasible weights in $\Lambda^{i}$. That is, the value function of agent $i$ is:

$$
v_{\Lambda^{i}}^{c}(a)=\min _{\lambda^{i} \in \Lambda^{i}} \sum_{j=1}^{s^{i}} \lambda_{j}^{i} u_{j}^{i}(a) .
$$


Note that since the sets of information are polyhedral and the minimum of a linear function on a polyhedron is reached at one of its extreme points, then the value function $v_{\Lambda^{i}}^{c}$ only depends on the extreme points of $\Lambda^{i}$. Formally, given the polyhedron of weights $\Lambda^{i} \subseteq \Delta^{s^{i}}$ with extreme points $\bar{\lambda}^{i}(r), r=1, \ldots, p^{i}$, the value function is

$$
v_{\Lambda^{i}}^{c}(a)=\min _{r=1, \ldots, p^{i}}\left\{v_{\bar{\lambda}(r)}^{i}(a)\right\}
$$

The decision rule consisting of the maximization of this value function constitutes the extension of the well-known max-min criterion (Wald, 1950) for the case of preference information. This extended rule is also formally related with the analysis of max-min expected utility developed by Gilboa and Schmeidler (1989).

In this conservative setting, the appropriate concept of equilibrium is the following.

Definition 3.4. An action profile $a^{*}=\left(a^{* 1}, \ldots, a^{* n}\right)$ is a conservative equilibrium for the game with preference information $\Lambda$ if $\nexists i \in N$ with $a^{i} \in A^{i}$ such that $v_{\Lambda^{i}}^{c}\left(a^{i}, a^{*-i}\right)>v_{\Lambda^{i}}^{c}\left(a^{*}\right)$.

$E_{\Lambda}^{c}(G)$ denotes the set of conservative equilibrium of the game $G$ with preference information $\Lambda$.

Equivalently, $a^{*} \in E_{\Lambda}^{c}(G)$ if for all $i \in N, v_{\Lambda^{i}}^{c}\left(a^{i}, a^{*-i}\right) \leq v_{\Lambda^{i}}^{c}\left(a^{*}\right)$, for all $a^{i} \in A^{i}$.

A first question to answer is whether conservative equilibria as defined above are equilibria for the game with preference information, as defined in 3.1. The answer is affirmative under the conditions stated in the following result.

Proposition 3.5. Let $G=\left\{\left(A^{i}, u^{i}\right)_{i \in N}\right\}$ be a game with vector-valued utilities. If $A^{i}$ is a non-empty convex subset of a finite dimensional space and, for each $i \in N$, $u^{i}$ is concave in $a^{i}$, then $E_{\Lambda}^{c}(G) \subseteq E_{\Lambda}(G)$.

Note that if functions $u_{j}^{i}$ are continuous then $v_{\Lambda^{i}}^{c}$ is continuous, although it is not differentiable in certain subsets of the strategy sets. Nevertheless, if functions $u_{j}^{i}$ are concave, then $v_{\Lambda^{i}}^{c}$ is also concave and the existence of a conservative equilibrium is guaranteed as a consequence of the well-known existence results.

\section{Mixed duopoly with various objectives}

In this section, we consider two firms with several objectives competing in the same industry. Each firm is primarily concerned with its own benefit, but considers a 
second goal. One of the firms is also a maximizer of the industry profit, i.e., it also takes into account the benefit of the industry as a whole. The other firm is a socially responsible firm that is concerned not only with its profit, but also with social welfare. In this sense, the second firm has features of a public firm and therefore we have a case within the so-called mixed duopoly models. Although the research in this type of duopoly goes back to Merrill and Schneider (1966), the expansion of the literature regarding this model starts in the 80's with the contribution of De Fraja and Del Bono (1989). The main innovation of our analysis with respect to the previous literature is that the social firm maintains the maximization of profits as one of its objectives.

The key element in the analysis is that the firm cannot compare in monetary terms the results obtained when it tries to achieve each of the two goals. An additional monetary unit of profit for the firm itself does not have the same meaning as an additional monetary unit in either the industrial profit or in social welfare, moreover, there is no clear way to aggregate their values. Therefore, the analysis of this mixed duopoly with firms trying to achieve various objectives is formalized in this section as a game with vector-valued utilities.

The two firms of the industry compete in quantities under Cournot assumptions and produce a homogeneous commodity. They face an inverse demand function given by $p=P(Q)$, where $Q=q^{1}+q^{2}$ is the total quantity produced in the market. Following the standard in the literature (see Kreps and Scheinkman, 1983), we assume that the function $P(Q)$ is twice-continuously differentiable, strictly decreasing, concave, and non-negative on some bounded interval $(0, K)$, and that $P(Q)=0$ for $Q \geq K$.

Firms are allowed to select any non-negative quantity. However, it could be argued that extremely large quantities are not feasible and so should not be included in a firm's strategy space. Therefore, the strategy spaces should be bounded. We also assume that each $A^{i}$ is closed. Thus, the strategy set of each firm is represented by a closed interval, $A^{i}=\left[0, k^{i}\right], i=1,2$. For simplicity, it is assumed that firms have no fixed costs and that their marginal costs are equal to zero. Under these assumptions, the quantity $K$ corresponds to the perfect competition quantity. In addition, we assume that the reservation price and market size are finite.

For $i=1,2$, the first objective for firm $i$ is its profit $u_{1}^{i}\left(q^{1}, q^{2}\right)=q^{i} P\left(q^{1}+q^{2}\right)$. Note that the profit function of each firm is strictly concave in its own action, since $\frac{\partial^{2} u_{1}^{i}}{\partial q^{i} \partial q^{i}}\left(q^{1}, q^{2}\right)<0$. As a consequence, given the action of one of the firms in the 
corresponding interval $\left(0, k^{i}\right)$, the profit of the other attains its maximum where its derivative is null. For $q^{j} \in\left(0, k^{i}\right)$, the optimal response function of firm $i$ to the action of firm $j$, denoted by $r_{1}^{i}\left(q^{j}\right)$, is implicitly defined by the following equation.

$$
P\left(q^{1}+q^{2}\right)+q^{i} P^{\prime}\left(q^{1}+q^{2}\right)=0 .
$$

For $q^{j} \geq K$, the optimal response function is defined as $r_{1}^{i}\left(q^{j}\right)=0$. Under our initial assumptions on the inverse demand functions, the reaction functions, $r_{1}^{i}$, are non-increasing and strictly decreasing over the range where it is strictly positive, and continuously differentiable (see Kreps and Scheikman, 1983). Moreover, these assumptions guarantee the existence of a unique Cournot equilibrium.

However, firms are not only concerned with their own profit. They consider an additional objective related to the welfare of the other economic agents, consumers and firms, and this also affects the development of the firm. Firm 1 is interested in the profit of the whole industry, defined as the joint benefit of the firms involved. A higher industrial benefit can be interpreted by the firm as a signal of a good performance of the industry, that could eventually have a positive impact on all firms. On the other hand, we have regarded Firm 2 as a socially responsible firm in the sense that it takes into account the welfare of all agents. Therefore, its second objective is social welfare which includes both the joint benefit of the two firms and the consumer surplus.

Since the objectives of Firm 1 are related to its own benefit and the industrial profit, the components of the utility of Firm 1 are its own benefit and the average benefit of the two firms that make up the industry, $u_{2}^{1}\left(q^{1}, q^{2}\right)=\frac{q^{1}+q^{2}}{2} P\left(q^{1}+q^{2}\right)$, and therefore, the vector-valued utility of Firm 1 is

$$
u^{1}\left(q^{1}, q^{2}\right)=\left(q^{1} P(Q), \frac{Q}{2} P(Q)\right)
$$

Firm 2 is concerned with its own benefit and with social welfare. The social welfare function is modeled as the sum of the joint benefit of the two firms and the consumer surplus. Thus, the function that evaluates social welfare is $u_{2}^{2}\left(q^{1}, q^{2}\right)=$ $C S\left(q^{1}+q^{2}\right)+\left(q^{1}+q^{2}\right) P\left(q^{1}+q^{2}\right)$. Therefore, the vector-valued utility of Firm 2 is

$$
u^{2}\left(q^{1}, q^{2}\right)=\left(q^{2} P(Q), C S(Q)+Q P(Q)\right)
$$

Under the assumptions on the inverse demand function, all the functions representing the objectives of each firm are strictly concave in the action of the corresponding firm. It is easy to see that $\frac{\partial^{2} u_{2}^{1}}{\partial q^{1} \partial q^{1}}\left(q^{1}, q^{2}\right)<0$, and that the optimal 


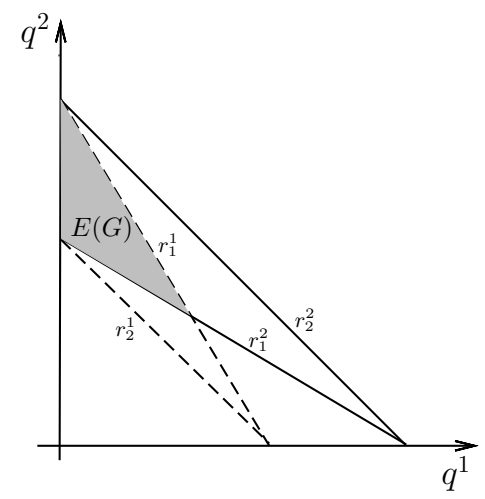

Figure 1: The set of equilibria of the mixed duopoly

response of Firm 1 to the actions of Firm 2, denoted $r_{2}^{1}\left(q^{2}\right)$, is $r_{2}^{1}\left(q^{2}\right)=Q_{M}-q^{2}$, where $Q_{M}$ is the monopoly quantity, that is to say, the quantity maximizing $Q P(Q)$.

Regarding the social welfare function, $u_{2}^{2}$, note that

$$
u_{2}^{2}\left(q^{1}, q^{2}\right)=C S\left(q^{1}+q^{2}\right)+\left(q^{1}+q^{2}\right) P\left(q^{1}+q^{2}\right)=\int_{0}^{q^{1}+q^{2}} P(x) d x .
$$

Therefore, $\frac{\partial u_{2}^{2}}{\partial q^{2}}\left(q^{1}, q^{2}\right)=P\left(q^{1}+q^{2}\right)$, and $\frac{\partial^{2} u_{2}^{2}}{\partial q^{2} \partial q^{2}}\left(q^{1}, q^{2}\right)=P^{\prime}\left(q^{1}+q^{2}\right)<0$. It follows that $u_{2}^{2}$ is also strictly concave in $q^{2}$. Since $\frac{\partial u_{2}^{2}}{\partial q^{2}}\left(q^{1}, q^{2}\right)=P\left(q^{1}+q^{2}\right)$, then the optimal response of Firm 2 to the actions of Firm 1, denoted $r_{2}^{2}\left(q^{1}\right)$, is $r_{2}^{2}\left(q^{1}\right)=Q_{P C}-q^{1}$, where $Q_{P C}$ is the perfect competition quantity.

It follows from Theorem 2.3 that the set of equilibria of the game with vectorvalued utilities representing the mixed duopoly, $G=\left\{\left(A^{i}, u^{i}\right)_{i=1,2}\right\}$, can be described in terms of the best responses as

$$
E(G)=\left\{\left(q^{1}, q^{2}\right): Q_{M}-q^{2} \leq q^{1} \leq r_{1}^{1}\left(q^{2}\right), r_{1}^{2}\left(q^{1}\right) \leq q^{2} \leq Q_{P C}-q^{1}\right\} .
$$

Note, that as a consequence of the strict concavity of the components of the utility, the set of equilibria coincides with the set of weak equilibria.

In order to simplify the presentation, we will graphically illustrate the results with examples in which the inverse demand functions are linear, that is $P(Q)=$ $a-b\left(q^{1}+q^{2}\right)$. The shaded area in Figure 1 shows the set of equilibria. Dashed lines represent the reaction function corresponding to the components of the utilities of Firm 1. Solid lines are the reactions of the components of the utility of Firm 2.

Since the equilibrium outcomes of the extended Cournot model must lie within this area, a wide range of action profiles cannot be equilibria. Note that the set of 
equilibria includes the original Cournot equilibrium. In any of the equilibria, the firm that maximizes industry profit will offer no more than what it would offer if it only considered its individual profit. By contrast, the socially responsible firm will always offer at least its Cournot quantity.

\subsection{An additional objective concerning market share}

In this subsection, we analyze a situation in which Firm 1, apart from the objectives considered before (individual profit and average benefit of both firms), also takes an objective concerning market share into account. Specifically, the firm wishes that its offer constitutes a certain proportion of the quantity offered by the other firm.

In order to formalize this additional objective, a new component for the utility of Firm 1 is defined ${ }^{2}, u_{3}^{1}\left(q^{1}, q^{2}\right)=-\left(q^{1}-\alpha q^{2}\right)^{2}$, for $\alpha>0$. The parameter $\alpha$ may have interesting interpretations. A firm strives to ensure a minimum market share against its competitor should select a value of $\alpha$ below one. A value of $\alpha$ greater than one could be interpreted as an entry deterrence strategy against potential competitors or as a signal of an expansive strategy of the firm, in an effort to achieve a dominant position in the market with the long-term advantages that this would entail.

For Firm 2, the objectives are the same as above. Thus, the corresponding vector-valued game is $\hat{G}=\left\{\left(A^{i}, \hat{u}^{i}\right)_{i=1,2}\right\}$, where

$$
\begin{gathered}
\hat{u}^{1}\left(q^{1}, q^{2}\right)=\left(q^{1} P(Q), \frac{Q}{2} P(Q),-\left(q^{1}-\alpha q^{2}\right)^{2}\right) . \\
\hat{u}^{2}\left(q^{1}, q^{2}\right)=\left(q^{2} P(Q), C S(Q)+Q P(Q)\right) .
\end{gathered}
$$

The best response function for the new component $u_{3}^{1}\left(q^{1}, q^{2}\right)=-\left(q^{1}-\alpha q^{2}\right)^{2}$ is $\hat{r}_{3}^{1}\left(q^{2}\right)=\alpha q^{2}$. Theorem 2.3 can also be applied in order to identify the expanded set of equilibria.

$$
E(\hat{G})=\left\{\left(q^{1}, q^{2}\right): \underline{r}^{i}\left(q^{j}\right) \leq q^{i} \leq \bar{r}^{i}\left(q^{j}\right), i=1,2\right\},
$$

with $\underline{r}^{1}\left(q^{2}\right)=\min _{j=1,2,3}\left\{r_{j}^{1}\left(q^{2}\right)\right\}, \bar{r}^{1}\left(q^{2}\right)=\max _{j=1,2,3}\left\{r_{j}^{1}\left(q^{2}\right)\right\}, \underline{r}^{2}\left(q^{1}\right)=\min _{j=1,2}\left\{r_{j}^{2}\left(q^{1}\right)\right\}$, and $\bar{r}^{2}\left(q^{1}\right)=\max _{j=1,2}\left\{r_{j}^{2}\left(q^{1}\right)\right\}$.

Figure 2 represents the expanded set of equilibria for the case of linear demand functions when $\alpha=1$, that is, when Firm 1 wishes to equalize the offer of Firm 2. The dashed line represents $\underline{r}^{1}\left(q^{2}\right)$ and the dotted line $\bar{r}^{1}\left(q^{2}\right)$. With the incorporation of this additional objective into the game, new equilibria emerge. In fact, in this

\footnotetext{
${ }^{2}$ Other representations of this objective exist. However, this form is convenient for our analysis.
} 


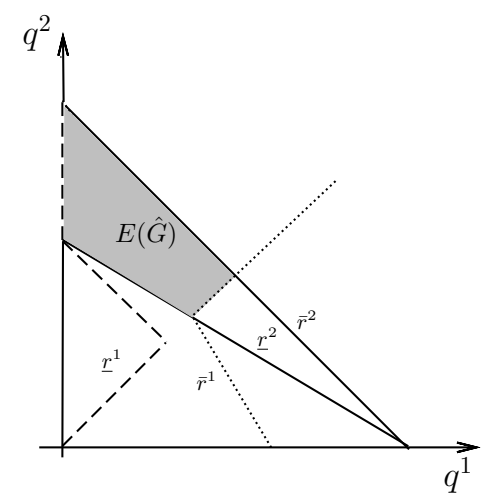

Figure 2: Equilibria of the mixed duopoly with an additional objective

new situation, it is obtained that, unlike the above case with two objectives, there are equilibria where both firms produce above the Cournot quantity.

\subsection{The mixed duopoly with preference information}

In this subsection, we turn back to our original mixed duopoly where each firm takes into account two objectives, $G=\left\{\left(A^{i}, u^{i}\right)_{i=1,2}\right\}$. We assume that there is information available about the relative importance of the objectives that the agents are considering.

In our model, we represent a situation in which Firm 1 gives more relevance to its first objective and therefore an additional unit of its own benefit is at least as important as an additional unit of average industrial profit. For Firm 2, the importance of its second objective is no less than that of the first objective, and thus an additional unit of social benefit is at least as relevant as an additional unit of individual benefit. This brings us closer to the traditional mixed oligopoly model, where there is a private firm that maximizes its own profit and a public firm that is only concerned about social welfare.

Formally, the information sets for Firm 1 and Firm 2 are respectively: $\Lambda^{1}=$ $\left\{\lambda^{1} \in \Delta^{2}: \lambda_{1}^{1} \geq \lambda_{2}^{1}\right\}, \Lambda^{2}=\left\{\lambda^{2} \in \Delta^{2}: \lambda_{2}^{2} \geq \lambda_{1}^{2}\right\}$. The extreme points of these sets of information are $(1,0),(1 / 2,1 / 2)$ for $\Lambda^{1}$ and $(1 / 2,1 / 2),(0,1)$ for $\Lambda^{2}$. Theorem 3.3 permits us to compute the set of equilibria of the game with partial information as the weak equilibria of a transformed game with vector-valued utilities $\left\{\left(A^{i}, v_{\Lambda}^{i}\right)_{i=1,2}\right\}$, where

$$
v_{\Lambda}^{1}\left(q^{1}, q^{2}\right)=\left(q^{1} P(Q), \frac{1}{4}\left(3 q^{1}+q^{2}\right) P(Q)\right) .
$$



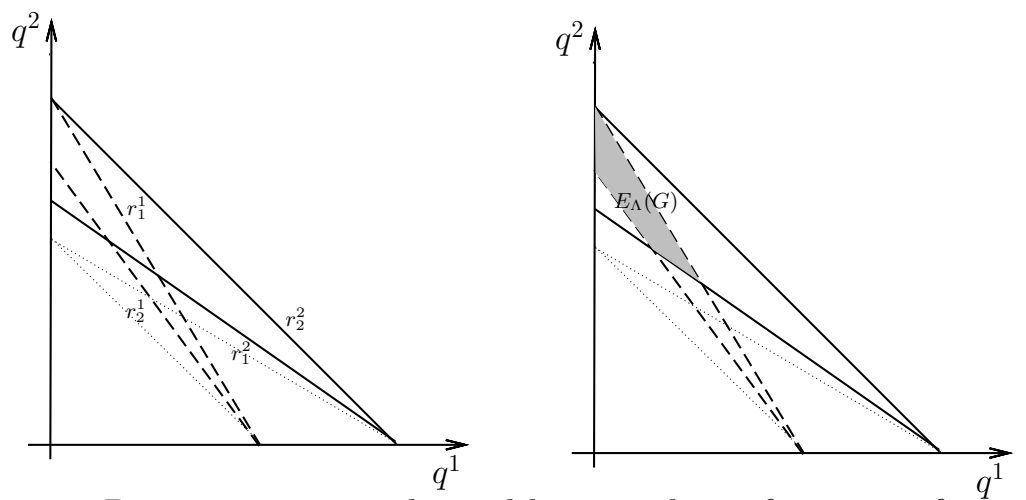

Figure 3: Reaction sets and equilibria with preference information

$$
v_{\Lambda}^{2}\left(q^{1}, q^{2}\right)=\left(\left(\frac{q^{1}}{2}+q^{2}\right) P(Q)+\frac{C S(Q)}{2}, C S(Q)+Q P(Q)\right) .
$$

Note that, since they consist of convex combinations of strictly concave functions, the components of this transformed game are also strictly concave in the action of the corresponding firm. Thus, the set of equilibria and the set of weak equilibria of the game $\left\{\left(A^{i}, v_{\Lambda}^{i}\right)_{i=1,2}\right\}$ coincide.

For the components of this transformed game, the best response functions are $\hat{r}_{1}^{1}\left(q^{2}\right)=Q_{M}-q^{2}$ and $\hat{r}_{2}^{2}\left(q^{1}\right)=Q_{P C}-q^{1}$ (they coincide with $r_{1}^{1}\left(q^{2}\right)$ and $r_{2}^{2}\left(q^{1}\right)$, respectively). On the other hand, $\hat{r}_{2}^{1}\left(q^{2}\right)$ and $\hat{r}_{1}^{2}\left(q^{1}\right)$ are obtained implicitly from

$$
3 P(Q)+\left(3 q^{1}+q^{2}\right) P^{\prime}(Q)=0, \quad 2 P(Q)+q^{2} P^{\prime}(Q)=0 .
$$

For the linear case, the new reaction functions and the set of equilibria are represented in Figure 3. Note that the new set of equilibria does not include the Cournot equilibrium. In comparison with the set of equilibria with no preference information, the quantity offered by Firm 1 is equal or less, and the quantity offered by Firm 2 is equal or greater.

In order to handle different degrees of importance for the components of the utilities, the information sets considered above can be generalized in the following sense: $\Lambda^{1}=\left\{\lambda^{1} \in \Delta^{2}: \lambda_{1}^{1} \geq \beta \lambda_{2}^{1}\right\}, \Lambda^{2}=\left\{\lambda^{2} \in \Delta^{2}: \lambda_{2}^{2} \geq \delta \lambda_{1}^{2}\right\}$, where $\beta, \delta \geq 0$. The extreme points are $(1,0)$ and $\left(\frac{\beta}{\beta+1}, \frac{1}{\beta+1}\right)$ for $\Lambda^{1}$, and $\left(\frac{1}{\delta+1}, \frac{\delta}{\delta+1}\right)$ and $(0,1)$ for $\Lambda^{2}$. The transformed game is defined by the following vector-valued functions:

$$
\begin{gathered}
v_{\Lambda}^{1}\left(q^{1}, q^{2}\right)=\left(q^{1} P(Q), \frac{1}{2(\beta+1)}\left((2 \beta+1) q^{1}+q^{2}\right) P(Q)\right) . \\
\left.v_{\Lambda}^{2}\left(q^{1}, q^{2}\right)=\left(q^{2}+\frac{\delta}{\delta+1} q^{1}\right) P(Q)+\delta C S(Q), C S(Q)+Q P(Q)\right) .
\end{gathered}
$$


For the case of linear demand, the reaction functions are $\hat{r}_{1}^{1}\left(q^{2}\right)=\frac{a}{2 b}-\frac{q^{2}}{2}, \hat{r}_{2}^{1}\left(q^{2}\right)=\frac{a}{2 b}-\frac{2 \beta+2}{2 \beta+1} q^{2}, \hat{r}_{1}^{2}\left(q^{1}\right)=\frac{a}{b} \frac{1+\delta}{2+\delta}-\frac{1+\delta}{2+\delta} q^{1}, \hat{r}_{2}^{2}\left(q^{2}\right)=\frac{a}{b}-q^{1}$.

The different values that the parameters can adopt determine the size of the set of equilibria. Note that the smaller the parameter, the wider the sets. For $\beta=0$ and $\delta=0$, the original set of equilibria, represented in Figure 1, is obtained. For high values of these parameters, the relevance of one of the objectives vanishes and the set of equilibria tends to be a singleton in which Firm 2 offers the perfect competition quantity and Firm 1 abandons the market.

\subsection{Conservative firms}

Another situation can arise when, apart from the information on preference weights, the firms show an attitude towards possible outcomes. We can assume that the firms adopt a conservative criterion, and, for each profile of strategies, they look at the worst weighted value from among those obtained with weights in its information set.

For the former mixed duopoly with preference information described by the sets $\Lambda^{1}=\left\{\lambda^{1} \in \Delta^{2}: \lambda_{1}^{1} \geq \lambda_{2}^{1}\right\}$ and $\Lambda^{2}=\left\{\lambda^{2} \in \Delta^{2}: \lambda_{2}^{2} \geq \lambda_{1}^{2}\right\}$, assume that the firms are conservative, in the sense that the evaluation of a pair of strategies consists of the worst weighted value from among those obtained with weights in $\Lambda^{1}$ and $\Lambda^{2}$. In this situation, the value function for Firm 1 is

$$
\begin{gathered}
v_{\Lambda}^{c 1}\left(q^{1}, q^{2}\right)=\operatorname{Min}\left\{q^{1} P(Q), \frac{1}{4}\left(3 q^{1}+q^{2}\right) P(Q)\right\} . \\
v_{\Lambda}^{c 1}\left(q^{1}, q^{2}\right)= \begin{cases}q^{1} P\left(q^{1}+q^{2}\right) & \text { when } q^{1} \leq q^{2} \\
\frac{1}{4}\left(3 q^{1}+q^{2}\right) P\left(q^{1}+q^{2}\right) & \text { when } q^{2} \leq q^{1} .\end{cases}
\end{gathered}
$$

This function is not differentiable when $q^{1}=q^{2}$. In order to describe the reaction function, the partition of the space of strategies has to be taken into account.

The conservative value function for Firm 2 is

$$
\begin{aligned}
v_{\Lambda}^{c 2}\left(q^{1}, q^{2}\right) & =\operatorname{Min}\left\{\left(\frac{q^{1}}{2}+q^{2}\right) P(Q)+\frac{C S(Q)}{2}, C S(Q)+Q P(Q)\right\}= \\
& =\left(\frac{q^{1}}{2}+q^{2}\right) P(Q)+\frac{C S(Q)}{2}, \text { for all }\left(q^{1}, q^{2}\right) .
\end{aligned}
$$




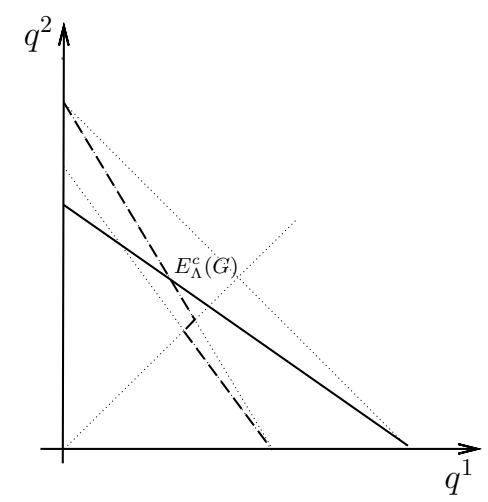

Figure 4: Conservative equilibrium with preference information

For the linear case, Figure 4 illustrates the reaction function of Firm 1 in dashed lines, the reaction function of Firm 2 in solid lines and the unique conservative equilibrium attained at $E_{\Lambda}^{c}(G)=\left\{\left(\frac{a}{4 b}, \frac{a}{2 b}\right)\right\}$. Note that the total equilibrium quantity is greater than the quantity offered when both firms are traditional profit maximizers.

For the generalized sets of weights $\Lambda^{1}=\left\{\lambda^{1} \in \Delta^{2}: \lambda_{1}^{1} \geq \beta \lambda_{2}^{1}\right\}, \Lambda^{2}=\left\{\lambda^{2} \in\right.$ $\left.\Delta^{2}: \lambda_{2}^{2} \geq \delta \lambda_{1}^{2}\right\}$, a unique conservative equilibrium always exists and is given by $\left(q^{1 *}, q^{2 *}\right)=\left(\frac{1}{3+\delta} \frac{a}{b}, \frac{1+\delta}{3+\delta} \frac{a}{b}\right)$. Note that in this case, the conservative equilibrium does not depend on the value of $\beta$, that is, the relevance of the first objective with respect to the second objective for Firm 1 does not affect the conservative equilibrium. When $\delta$ tends towards zero, the conservative equilibrium becomes the Cournot equilibrium. As $\delta$ increases, the total quantity offered in the market tends to be the perfect competition quantity and is only provided by Firm 2 .

\section{Conclusions}

We present an innovative analysis of non-cooperative games with vector-valued utility functions. These games have a high potential to represent situations in which the agents act strategically in order to achieve several goals simultaneously. We have shown in the paper that this model permits us to accommodate partial information about the incomplete preferences of the agents in order to attain predictions of the results of the interaction of a more realistic nature.

Our first result is interesting since it characterizes the whole set of equilibria for a wide class of these games in terms of the reaction functions of the components of the vector-utility of the agents, in a similar way to what happens in the case of 
scalar non-cooperative games. This result also facilitates the identification of the set of equilibria when the agents provide information about their preferences.

The increase of the dimensionality of the agents' utilities causes new equilibria to arise, therefore the possibility of refining the set of equilibria becomes a relevant issue. We focus on the cases in which preferential information consists of sets of weights described by linear relations. This way of representing information is easily understood by the agents and is convenient from the operational point of view. In this sense, our second result identifies the set of equilibria of the vector-valued game with preference information with the set of equilibria of a transformed game, which, in general, is also a vector-valued game.

The results are applied to the analysis of a type of mixed duopoly where the firms consider different social goals but do not completely abandon the pursuit of maximum benefit which ensures their permanence in the market. The addition of the new goals expands the set of equilibria. Nevertheless, the incorporation of preference information and of possible attitudes that can be adopted by the firms allows us to obtain equilibria in accordance with the information provided. Since a conservative attitude of the agents is usual in many economic situations, our approach also explores the possibility of the adoption of a conservative criterion in order to identify the corresponding equilibria. In this context, for conservative firms, the equilibrium is unique and it only depends on the relevance that the social firm gives to each objective.

Our approach is flexible and provides a useful theoretical framework for the analysis of various situations in the goods and factors markets where economic agents wish to attain several goals. This is the case for responsible consumers, who take into account not only their own welfare but also the social and environmental impact of their consumption decisions. Moreover, in the labour market, the approach presented in this paper could be helpful in modeling the behavior of trade unions, which have objectives related to both the welfare of the workers and also to the welfare of the institution.

Acknowledgements: The research of the authors is partially supported by the Spanish Ministry of Economy and Competitiveness, project ECO2015-68856-P (MINECO/FEDER) . 


\section{Appendix}

Proof Theorem 2.3. First, note that under the assumptions, all the values $r_{j}^{i}\left(a^{-i}\right)$ are well-defined. Let $a \in \times_{i \in N} A^{i}$ be a profile of strategies such that for a certain $i \in N, a^{i}<\underline{r}^{i}\left(a^{-i}\right)$. Since $u_{j}^{i}$ is strictly concave in its own action, then at $a^{i}, u_{j}^{i}$ is strictly increasing for all $j \in J^{i}$, and by adopting a strategy $a^{i}+\varepsilon$, with $\varepsilon>0$, agent $i$ will increase all the components of his utility. Therefore, $a$ is not an equilibrium. Analogous reasoning can be applied when $a^{i}>\bar{r}^{i}\left(a^{-i}\right)$. Let $a \in \times_{i \in N} A^{i}$, such that $\underline{r}^{i}\left(a^{-i}\right) \leq a^{i} \leq \bar{r}^{i}\left(a^{-i}\right)$ for all $i \in N$. For $i \in N$, if $\underline{r}^{i}\left(a^{-i}\right)=a^{i}=\bar{r}^{i}\left(a^{-i}\right)$, by moving from his strategy, all the components of the utility of agent $i$ will decrease. If $\underline{r}^{i}\left(a^{-i}\right)<a^{i}<\bar{r}^{i}\left(a^{-i}\right)$, at least one of the inequalities in $\underline{r}^{i}\left(a^{-i}\right) \leq a^{i} \leq \bar{r}^{i}\left(a^{-i}\right)$ must be strict. If $\underline{r}^{i}\left(a^{-i}\right)<a^{i}$, by reducing $a^{i}$, the utility corresponding to $\bar{r}^{i}$ will decrease. If $a^{i}<\bar{r}^{i}\left(a^{-i}\right)$, by increasing $a^{i}$, the utility corresponding to $\underline{r}^{i}$ will decrease.

Proof Theorem 2.5. A first inclusion is proved in Wang (1993). Conversely, let $a^{*} \in E^{w}(G)$. For $i \in N$, define the sets $Y^{i}=\left\{x \in \mathbb{R}^{s^{i}}: u^{i}\left(a^{i}, a^{*-i}\right) \geqq\right.$ $x$, for some $\left.a^{i} \in A^{i}\right\}$, and $X^{i}=\left\{x \in \mathbb{R}^{s^{i}}: u^{i}\left(a^{*}\right)<x\right\}$. The sets $Y^{i}$ and $X^{i}$ are convex and disjoint. Convexity is a consequence of the concavity of $u^{i}$. To prove that they are disjoint: If $x \in Y^{i}$ then $a^{i} \in A^{i}$ exists such that $x \leqq u^{i}\left(a^{i}, a^{*-i}\right)$. If $x \in X^{i}$ then $x>u^{i}\left(a^{*}\right)$. Therefore, if $x \in Y^{i} \cap X^{i}$, then $u^{i}\left(a^{*}\right)<x \leqq u^{i}\left(a^{i}, a^{*-i}\right)$, and $a^{i} \in A^{i}$ exists with $u^{i}\left(a^{*}\right)<u^{i}\left(a^{i}, a^{*-i}\right)$, which contradicts $a^{*}$ being a weak equilibrium of $G$. It follows from Minkowski's separating hyperplane theorem that there exists some non-null vector $\lambda^{i} \in \mathbb{R}^{s^{i}}$ and some constant $c$ such that $\lambda^{i} \cdot x \leq c$ for all $x \in Y^{i}$, and $\lambda^{i} \cdot x \geq c$ for all $x \in X^{i}$. It is easy to see that a non-negative $\lambda^{i}$ and $c=\lambda^{i} \cdot u^{i}\left(a^{*}\right)$ satisfy this condition. Therefore, for each $i \in N, \lambda^{i} \cdot u^{i}\left(a^{*}\right) \geq \lambda^{i} \cdot x$ for all $x \in Y^{i}$, and since $u^{i}\left(a^{i}, a^{*-i}\right) \in Y^{i}$, for all $a^{i} \in A^{i}, a^{* i}$ maximizes $v_{\lambda}^{i}\left(a^{i}, a^{*-i}\right)$. Since only the direction of the vector matters, $\lambda^{i}$ can be taken in $\Delta^{s^{i}}$. It follows that $a^{*} \in E\left(G_{\lambda}\right)$.

Proof Proposition 3.2. If $a^{*} \in E_{\Lambda}(G)$, then for each $i \in N$ there exists $\lambda^{i} \in \Lambda^{i}$ such that $a^{*}$ is an equilibrium of game $G_{\lambda}$. It follows from Definition 3.1 that $\lambda^{i} \cdot u^{i}\left(a^{*}\right) \geq \lambda^{i} \cdot u^{i}\left(a^{i}, a^{*-i}\right)$, for all $a^{i} \in A^{i}$. On the other hand, for each $i \in N, \lambda^{i} \in \Lambda^{i}$ can be written as a convex combination of the extreme points of $\Lambda^{i}, \lambda^{i}=\sum_{r=1}^{p^{i}} \alpha_{r}^{i} \bar{\lambda}^{i}(r)$ with $\alpha_{r}^{i} \geq 0$, and $\sum_{r=1}^{p^{i}} \alpha_{r}^{i}=1$. Thus, for each $i \in N$, $\sum_{r=1}^{p^{i}} \alpha_{r}^{i} \bar{\lambda}^{i}(r) \cdot u^{i}\left(a^{*}\right) \geq \sum_{r=1}^{p^{i}} \alpha_{r}^{i} \bar{\lambda}^{i}(r) \cdot u^{i}\left(a^{i}, a^{*-i}\right)$ for all $a^{i} \in A^{i}$. It follows that $a^{*}$ is a weak equilibrium of game $\left\{\left(A^{i}, v_{\Lambda}^{i}\right)_{i \in N}\right\}$. 
Proof Theorem 3.3. A first inclusion is stated in Proposition 3.2. Conversely, first note that, if for each $i, u^{i}$ is concave in $a^{i}$, then, $v_{\Lambda}^{i}$ is also concave. Let $a^{*}$ be a weak equilibrium of game $\left\{\left(A^{i}, v_{\Lambda}^{i}\right)_{i \in N}\right\}$. It follows from Theorem 2.5 that for each $i \in N, \alpha^{i} \in \Delta^{p^{i}}$ exists such that $\sum_{r=1}^{p^{i}} \alpha_{r}^{i} \bar{\lambda}^{i}(r) \cdot u^{i}\left(a^{*}\right) \geq \sum_{r=1}^{p^{i}} \alpha_{r}^{i} \bar{\lambda}^{i}(r) \cdot u^{i}\left(a^{i}, a^{*-i}\right)$ for all $a^{i} \in A^{i}$. Since $\sum_{r=1}^{p^{i}} \alpha_{r}^{i} \bar{\lambda}^{i}(r) \in \Lambda^{i}$, from Definition 3.1 it follows that $a^{*} \in E_{\Lambda}(G)$.

Proof Proposition 3.5. Let $a^{*} \in E_{\Lambda}^{c}(G)$ with $\Lambda=\times_{i \in N} \Lambda^{i}$. Thus, $\nexists i \in N$ with $a^{i} \in A^{i}$ such that $\min _{r=1, \ldots, p^{i}}\left\{v_{\bar{\lambda}(r)}^{i}\left(a^{i}, a^{*-i}\right)\right\}>\min _{r=1, \ldots, p^{i}}\left\{v_{\bar{\lambda}(r)}^{i}\left(a^{*}\right)\right\}$, where $\bar{\lambda}^{i}(r), r=1, \ldots, p^{i}$ are the extreme points of $\Lambda^{i}$. Suppose on the contrary that $a^{*} \notin E_{\Lambda}(G)$. Under convexity assumptions, by Theorem 3.3, $E_{\Lambda}(G)=E^{w}\left(G_{\Lambda}\right)$. Hence, $a^{*}$ is not a weak equilibrium of the game $\left\{\left(A^{i}, v_{\Lambda}^{i}\right)_{i \in N}\right\}$. That is, $\exists i \in N$ with $a^{i} \in A^{i}$ such that $v_{\Lambda}^{i}\left(a^{i}, a^{*-i}\right)>v_{\Lambda}^{i}\left(a^{*}\right)$. The components of $v_{\Lambda}^{i}$ are $v_{\bar{\lambda}(r)}^{i}$ for $i=1, \ldots, p^{i}$, and therefore $v_{\bar{\lambda}(r)}^{i}\left(a^{i}, a^{*-i}\right)>v_{\bar{\lambda}(r)}^{i}\left(a^{*}\right), \forall r=1, \ldots, p^{i}$. It follows that $\min _{r=1, \ldots, p^{i}}\left\{v_{\bar{\lambda}(r)}^{i}\left(a^{i}, a^{*-i}\right)\right\}>\min _{r=1, \ldots, p^{i}}\left\{v_{\bar{\lambda}(r)}^{i}\left(a^{*}\right)\right\}$, and this contradicts that $a^{*}$ is a conservative equilibrium for the game with preference information $\Lambda$.

\section{$7 \quad$ References}

Aumann, R. (1962) Utility theory without the completeness axiom. Econometrica 30, 445-462.

Bade, S. (2005) Nash equilibrium in games with incomplete preferences. Economic Theory 26, 309-332.

Bewley, T. (1986) Knightian utility theory: Part 1. Cowles Foundation Discussion Paper 807.

Blackwell, D. (1956) An analog of the minimax theorem for vector payoffs. Pacific Journal of Mathematics, 1-8.

Borm, P.E.M., Tijs, S.H., Van Den Aarssen, J. C. M. (1988) Pareto equilibria in multiobjective games. Methods of Operations Research 60, 303-312.

Corley, H.W. (1985) Games with vector payoffs. Journal of Optimization Theory and Applications 47, 491-498.

De Fraja G., Del Bono, F. (1989) Alternative strategies of a public enterprise in oligopoly. Oxford Economic Papers 41, 302-331. 
De Fraja G., Del Bono, F. (1990) Game theoretic models of mixed oligopoly. Journal of Economic Surveys 4, 1-7.

Dubra, J., Maccheroni, F., Ok, E. (2004) Expected utility theory without the completeness axiom. Journal of Economic Theory 115, 118-133.

Gilboa, I., Schmeidler, D. (1989) Maxmin expected utility with non-unique prior. Journal of Mathematical Economics 18, 141-153.

Keeney, R. L., Raiffa, H. (1976) Decisions with multiple objectives: preferences and value tradeoffs. Wiley. New York.

Kozhan, R., Salmon, M. (2009) Uncertainty aversion in a heterogeneous agent model of foreign exchange rate formation. Journal of Economic Dynamics \& Control 33, 1106-1122.

Kreps, D.M., Scheinkman, J.A. (1983) Quantity precommitment and Bertrand competition yield Cournot outcomes. The Bell Journal of Economics 14, 326337.

Merrill, W., N. Schneider (1966) Government firms in oligopoly industries: A short run analysis. Quarterly Journal of Economics 80, 400-12.

Nash, J. (1951) Non-cooperative games. Annals of Mathematics, 54(2), 286-295

Ok, E.A. (2002) Utility representation of an incomplete preference relation. Journal of Economic Theory 104, 429-449.

Park, J. (2015) Potential games with incomplete preferences. Journal of Mathematical Economics 61, 58-66.

Patriche, M. (2014) Existence of equilibrium for multiobjective games in abstract convex spaces. Mathematical Reports 16, 243-252.

Sagi, J.S. (2006) Anchored preference relations. Journal of Economic Theory 13(1), 283-295.

Shafer, W., Sonnenschein, H. (1975) Equilibrium in abstract economies without ordered preferences. Journal of Mathematical Economics 2, 345-348.

Shapley, L.S. (1959) Equilibrium points in games with vector payoffs. Naval Research Logistics Quarterly 6(1), 57-61. 
Voorneveld, M., Vermeulen, D., Borm, P. (1999) Axiomatizations of Pareto Equilibria in Multicriteria Games. Games and Economic Behavior 28, 146-154.

Wakker, P. (2001) Testing and characterizing properties of nonadditive measures through violations of the sure-thing principle. Econometrica 69(4),1039-1059.

Wald, A. (1950) Statistical Decision Functions. Wiley. New York.

Wang, S.Y. (1993) Existence of Pareto equilibrium. Journal of Optimization Theory and Applications 79, 373-384.

Zeleny, M. (1975) Games with multiple payoff. International Journal of Game Theory 4(4), 179-191.

Zhao, J. (1991) The equilibria of multiple objective games. The International Journal of Game Theory 20, 171-182. 